Research Article

\title{
Cross Cultural Adaptation and Validation of Italian Version of the Leeds Assessment of Neuropathic Symptoms and Signs Scale and Pain DETECT Questionnaire for the Distinction between Nociceptive and Neuropathic Pain
}

\author{
Alberto Migliore, ${ }^{1}$ Gianfranco Gigliucci, ${ }^{1}$ Antimo Moretti ${ }^{\mathbb{D}},{ }^{2}$ Alessio Pietrella, \\ Marco Peresson, ${ }^{3}$ Fabiola Atzeni, ${ }^{4}$ Piercarlo Sarzi-Puttini, ${ }^{5}$ Laura Bazzichi, ${ }^{6}$ \\ Sara Liguori $(\mathbb{D})^{2}$ and Giovanni Iolascon ${ }^{2}$ \\ ${ }^{1}$ Rheumatology Unit, San Pietro Fatebenefratelli Hospital, Rome, Italy \\ ${ }^{2}$ Department of Medical and Surgical Specialties and Dentistry, University of Campania "Luigi Vanvitelli", Naples, Italy \\ ${ }^{3}$ Neurological Section, San Pietro Fatebenefratelli Hospital, Rome, Italy \\ ${ }^{4}$ Rheumatology Unit, University of Messina, Messina, Italy \\ ${ }^{5}$ Rheumatology Unit, ASST-Fatebenefratelli-L. Sacco University Hospital, Milan, Italy \\ ${ }^{6}$ Rheumatology Unit, Department of Clinical and Experimental Medicine, University of Pisa, Pisa, Italy
}

Correspondence should be addressed to Antimo Moretti; antimo.moretti@unicampania.it

Received 22 December 2020; Revised 12 March 2021; Accepted 19 April 2021; Published 29 April 2021

Academic Editor: Parisa Gazerani

Copyright (c) 2021 Alberto Migliore et al. This is an open access article distributed under the Creative Commons Attribution License, which permits unrestricted use, distribution, and reproduction in any medium, provided the original work is properly cited.

Objective. This study aimed to validate Italian versions of Leeds Assessment of Neuropathic Symptoms and Signs (LANSS) scale and Pain DETECT questionnaire (PD-Q) and evaluate the ability of these questionnaires to discriminate between nociceptive and neuropathic pain. Design. Multicenter prospective validation cohort study. Subjects and Setting. One hundred patients were included with a diagnosis formulated by a specialist in outpatient settings (50 affected by knee osteoarthritis as nociceptive pain and 50 affected by trigeminal or postherpetic neuralgia as neuropathic pain). Methods. The Italian versions of both questionnaires according to Italian cultural characteristics were performed according to the following steps: (1) translation of the questionnaires from English into Italian; (2) review by a bilingual individual for consistency; (3) proposed version after a mail round between experts; (4) backward translation; (5) comparison with the original English version by the experts; (6) approved version of the questionnaires. One hundred patients were enrolled and completed the two questionnaires administered by a specialist or blinded nursing staff, at the baseline and after 24/48 hours. Internal consistency, stability, validity, and discriminative power were analyzed. Results. Statistically significant differences were reported about the ability of both questionnaires to discriminate between patients affected by neuropathic or nociceptive pain. Internal consistency for the Italian version of the LANSS was 0.76 , and for PD-Q, it was 0.80 , assessed by Cronbach's $\alpha$; LANSS showed a good test-retest reliability with an ICC of 0.76 , and PD-Q showed a high test-retest reliability with an ICC of 0.96 . For interrater reliability, there was a concordance rate of $83.3 \%$ between reference diagnosis and LANSS (Cohen's kappa $=0.67$, CI 95\% 0.52-0.75). Conclusions. This study validated the Italian versions of LANSS and PD-Q as reliable instruments with good psychometric characteristics, for pain evaluation, discriminating between nociceptive and neuropathic pain. Our findings were similar to those observed in the original study. Furthermore, we have reported the test-retest reliability for both questionnaires, not addressed in original validation studies. 


\section{Introduction}

Pain treatment is a key element in the management of many chronic diseases. In this regard, traditional therapeutic strategies addressed pain intensity, while, recently, a more appropriate approach taking into account the pathogenic mechanisms of pain has been widely used [1]. However, in clinical practice, many analgesic drugs are still prescribed without adopting pathogenic criteria with a consequent increased risk of subtherapeutic responses [2].

This is especially true when it comes to treating neuropathic or mixed pain. To correctly identify patients who can respond to a specific intervention, validated assessment tools have been produced. In particular, several questionnaires have been created and validated in different languages to discriminate between different types of pain, such as the Pain DETECT questionnaire (PD-Q) and the Leeds Assessment of Neuropathic Symptoms and Signs (LANSS) scale [3, 4], which can be used in the challenging diagnosis of neuropathic pain $(\mathrm{NeP})$. Nowadays, an Italian version of these questionnaires is not available; their translations and cross-cultural adaptations might be useful for Italian physicians to improve the management of different types of pain in clinical practice.

The objectives of this study were to translate and validate Italian versions of the LANSS and PD-Q scales, as well as to investigate the ability of these tools to discriminate between inflammatory/mechanical (nociceptive) and $\mathrm{NeP}$.

\section{Methods}

A multicenter prospective cohort study was conducted with an overall duration of 8 months (4 months of observation, 3 months of enrollment, and 1 month of data compilation), involving 5 outpatient services belonging to the Italian Society for Unified and Interdisciplinary Management of Musculoskeletal Pain and Algodystrophy (Società Italiana per la Gestione Unificata e Interdisciplinare del Dolore muscolo-scheletrico e dell'Algodistrofia, SI-GUIDA) for the inclusion of 100 patients.

Italian versions of both questionnaires were first developed through translation and back-translation and then analyzed for internal consistency, stability, validity, and discriminative power.

The guidelines of Beaton et al. [5] were used for validation, applying a translation and cultural adaptation. A first translation of the questionnaires was performed from the original English versions according to Italian cultural characteristics by three medical translators, reviewed by a bilingual individual to evaluate conceptual errors or inconsistencies. The proposed version of the questionnaires was revised in a mail round between experts involved in this study. Then, two English native speakers, with no access to the original version, performed backward translations. New versions were compared to the original ones by an expert involved in the study, and then, approved version of the questionnaires was produced. Finally, the definitive Italian version was validated in a clinical setting.

In this study, we considered three paradigmatic diseases causing nociceptive or neuropathic pain, namely, osteoarthritis, and trigeminal or postherpetic neuralgia, respectively.

A series of 100 subjects, of which 50 with nociceptive pain and 50 with $\mathrm{NeP}$ were included. Sociodemographic information on the patient and data about the general characteristics of the index disease was collected (date of diagnosis and of symptoms onset, and clinical significance). The patients enrolled in the validation study were selected by the specialist of the different centers in the routine clinical practice. Inclusion criteria were age $\geq 18$ years; diagnosis of trigeminal neuralgia or postherpetic neuralgia ( $\mathrm{NeP}$ group) or a diagnosis of knee osteoarthritis (OA) according to American College of Rheumatology (ACR) criteria [6] (nociceptive pain group), for at least 3 months; pain intensity assessed according to Visual Analogue Scale (VAS) at baseline $\geq 40 \mathrm{~mm}$ and persistence of symptoms for at least 3 months; complete ability to understand and speak the Italian language; patients able to adhere to the study procedures; patients able to understand and sign informed consent. Exclusion criteria were adults with mixed features of pain; cancer pain or fibromyalgia; presence of other joint diseases (rheumatoid arthritis; spondyloarthritis; connectivitis; polymyalgia rheumatica; gout; Paget's disease of bone; history of septic arthritis; fractures; osteonecrosis); patients taking analgesics or intra-articular treatments; impaired cognitive status; any sensory impairment that may interfere with the compilation of the questionnaire (blindness, deafness); poor or inadequate ability to understand and speak Italian language.

The diagnosis formulated by the specialist was considered as the "reference diagnosis" for the validation of the questionnaires. At the end of the specialist visit, the patient was asked to participate in the study. In case of acceptance, the patient signed an informed consent in order to be included in the study and to allow the use of the collected data. The study protocol and informed consent forms of the study were submitted to and approved by the local ethics committee, in accordance with the ethical principles originating from the Declaration of Helsinki. The questionnaires were administered to patients selected by another specialist or blinded nursing staff. Each patient filled out the questionnaires twice, at baseline and after $24-48$ hours. In addition, the personnel who administered the questionnaires had to answer a series of questions about the time needed to complete the questionnaire, the patient's ability to answer without help, and the grade of difficulty in understanding each item. A note was made to report difficulties encountered by each patient. The results of each questionnaire were calculated according to the original scoring system and assessed taking into account the "reference diagnosis" formulated during the baseline visit.

To minimize the risk of short-term clinical change, treatment of these patients remained constant during the study period.

\subsection{Outcome Measures}

2.1.1. LANSS Scale. The LANSS scale is a brief and easily applied instrument including the assessment of 5 symptoms and 2 signs. It is a semistructured interview, in which the 
patient is asked whether the description presented matches the pattern of pain felt during the past week. The original version was tested and validated in several settings with sensitivity ranging between $82 \%$ and $91 \%$ and specificity ranging from $80 \%$ to $94 \%$ compared with clinical diagnosis [4].

The LANSS includes a total of 7 items grouped in 2 sections. The first section (Section A) consists of 5 questions scored from 1 to 5 depending on the items whenever the answer is affirmative, and 0 if this is absent. The following descriptors are most frequently used by patients with NeP: bursting, electric shocks, changes in skin temperature/color, and others. It is the only neuropathic screening that investigates autonomic changes [7]. Section $B$ refers to the physical examination, in which the sensorial characteristics of pain, such as allodynia and hyperalgesia, are explored by means of skin stimulation (stroking cotton wool and pinprick): score is 0 if the answer is negative, and 5 or 3 , depending on the question, if positive. Therefore, the sum of all points obtained on the different items of both sections may vary between 0 and 24, and a cutoff point of 12 has been set as being indicative of $\mathrm{NeP}$.

2.1.2. $P D-Q$. The $\mathrm{PD}-\mathrm{Q}$ was originally developed for people with low-back pain and showed good sensitivity (85\%) and specificity (80\%) when compared to clinical diagnosis of a predominantly nociceptive (e.g., visceral-pain) or neuropathic (e.g., postherpetic neuralgia) origin [3]. The PD-Q classifies people into different groups according to a summative score from nine-items: $\mathrm{NeP}$ component is unlikely $(\leq 12)$, result is ambiguous [8-13], and $\mathrm{NeP}$ component is likely $(\geq 19)$. Most items use a 6-point scale, where higher scores are suggestive of greater intensity.

2.2. Statistical Analysis. Statistical analyses were performed using SPSS version 15.0 for Windows (Statistical Package for Social Sciences. Inc., Chicago, IL, USA). All continuous data were described by mean \pm standard deviation and qualitative data by percentage. The significance of the differences between continuous variables was analyzed using the one-way analysis of variance (ANOVA) and Student's $t$-test (two independent sample $t$-tests were used), while categorical variables were analyzed with chi-squared test.

For reliability analysis, Cronbach's $\alpha$ was used to assess the internal consistency [14], and intraclass correlation coefficient (ICC) between test and retest scores was used to assess stability over time [15]. The correlation between the assessment tools (PD-Q and LANSS) and clinical diagnosis was calculated using Pearson's correlation coefficient, and diagnostic classification of patients according to questionnaires was compared with clinical judgment.

In all analyses, $p$ values $<0.05$ were considered statistically significant.

\section{Results}

The study involved 100 patients, 50 patients affected by NeP (trigeminal or postherpetic neuralgia) (15 men and 35 women, mean age 50 years, range $21-85$ years) and 50 patients affected by nociceptive pain (knee OA) (20 men and 30 women, mean age 60 years, range $50-70$ years) (see Table 1 for further details).

The Italian translations of the questionnaires corresponded extremely well to the original versions, and no problems were reported by patients in the compilation. Questions results were clear and relevant for the description of pain.

Analyzing the psychometric properties of both questionnaires, all items of LANSS scale showed a strong factor loading with the first factor (ranging from 0.65 to 0.96 ). Cronbach's alpha value was 0.76 for the total LANSS score. Regarding LANSS stability, 100 patients have been retested within 48 hours, and the scores have shown high stability $(\mathrm{ICC}=0.76)$. For the validity of the LANSS scale, using a cutoff $\geq 12$, we obtained a sensitivity of $87 \%$ and specificity of $72 \%$; the overall classification was $82 \%$, positive predictive value (PPV) was $83 \%$, and negative predictive value (NPV) was $79 \%$. The diagnostic concordance rate between the clinical diagnosis and the LANSS scale was $83.3 \%$ (Cohen's kappa $=0.67$, CI 95\% 0.52-0.75).

For the validity of PD-Q, we obtained a sensitivity of $85 \%$ and specificity of $75 \%$; the overall classification was $81 \%$, positive predictive value (PPV) was $82 \%$, and negative predictive value (NPV) was $76 \%$. The diagnostic concordance rate between the clinical diagnosis and the PD-Q was 83.5\% (Cohen's kappa $=0.68$, CI 95\% 0.54-0.75).

The internal consistency of the Italian version of PD-Q was 0.80 assessed by Cronbach's $\alpha$. The mean overall score of the PD-Q was $16.33 \pm 8.35$ at baseline and $15.9 \pm 8.17$ when performing the retest measurement. The PD-Q test-retest reliability had a result of 0.96 for the total score, ranging from $0.84-0.96$ for individual items. No floor or ceiling effect was observed.

Statistically significant differences were reported about the capability of the questionnaires to differentiate patients affected by neuropathic or nociceptive pain, without differences in the same type of pain between the results reported at baseline and at retest (Table 2). Specifically, as reported in Table 2, according to pain reference diagnosis, $\mathrm{NeP}$ group showed a mean PD-Q score of $22.35 \pm 4.8$ (retest $21.5 \pm 4.1$ ), while NoP group reported a mean score of $4.8 \pm 3.7$ (retest 5.2 \pm 3.5 ), with a cutoff suggestive of $\mathrm{NeP}$ of $\geq 19$. Regarding the LANSS score, $\mathrm{NeP}$ group showed a mean score of $18 \pm 4.8$ (retest $17.5 \pm 4.6$ ), while NoP group reported a mean value of $4.4 \pm 3.4$ (retest $4.5 \pm 3.7$ ), with a cutoff point of 12 as indicative of NeP. Table 3 shows the results of $\mathrm{PD}-\mathrm{Q}$ in the $\mathrm{NeP}$ and the nociceptive pain group, respectively.

\section{Discussion}

This study was designed to validate the Italian versions of LANSS and PD-Q by analyzing the psychometric properties of these questionnaires and to define the ability of these diagnostic tools in discriminating between nociceptive and $\mathrm{NeP}$.

Our results demonstrated a correspondence of Italian translations of LANSS and PD-Q to the original versions; 
TABLE 1: Baseline demographic data according to pain reference diagnosis.

\begin{tabular}{lcc}
\hline & NoP group $(N=50)$ & NeP group $(N=50)$ \\
\hline Gender, $N(\%)$ & & $35(70 \%)$ \\
$\quad$ Women & $30(60 \%)$ & $15(30 \%)$ \\
Men & $20(40 \%)$ & $50 \pm 19$ \\
Age (years-mean \pm SD) & $60 \pm 6$ & $70 \pm 9$ \\
Weight (Kg-mean \pm SD) & $78 \pm 15$ & $165 \pm 12$ \\
Height $(\mathrm{cm}-$-mean \pm SD) & $168 \pm 8$ & $26 \pm 3$ \\
BMI (Kg/m2-mean \pm SD) & $28 \pm 7$ & $20(40 \%)$ \\
Education level, $N(\%)$ & $24(48 \%)$ & $18(36 \%)$ \\
$\quad$ Primary & $16(32 \%)$ & $12(24 \%)$ \\
High & $10(20 \%)$ & \\
$\quad$ University & & \\
\hline
\end{tabular}

Descriptive data for continuous variables are expressed as mean \pm standard deviation (SD); discrete variables are expressed as numbers $(N)$ and percentages (\%). NoP: nociceptive pain; NeP: neuropathic pain; BMI: body mass index.

TABLE 2: VAS score, LANSS score (test/retest), and PD-Q score (test/retest) according to pain reference diagnosis.

\begin{tabular}{|c|c|c|c|c|c|c|c|}
\hline & VAS & PD-Q score (test) & PD-Q score (retest) & $p$ value* & LANSS score (test) & LANSS score (retest) & $p$ value ${ }^{*}$ \\
\hline $\mathrm{NeP}$ group $(N=50)$ & $6.8 \pm 2.9$ & $22.35 \pm 4.8$ & $21.5 \pm 4.1$ & $>0.05$ & $18 \pm 4.8$ & $17.5 \pm 4.6$ & $>0.05$ \\
\hline NoP group $(N=50)$ & $6.3 \pm 2.3$ & $4.8 \pm 3.7$ & $5.2 \pm 3.5$ & $>0.05$ & $4.4 \pm 3.4$ & $4.5 \pm 3.7$ & $>0.05$ \\
\hline$p$ value $^{*}$ & $>0.05$ & $<0.01$ & $<0.01$ & & $<0.01$ & $<0.01$ & \\
\hline
\end{tabular}

Descriptive data for continuous variables are expressed as mean \pm standard deviation (SD); discrete variables are expressed as numbers $(N) ;{ }^{*} p$ values $<0.05$ were considered statistically significant. NeP: Neuropathic pain; NoP: nociceptive pain; VAS: visual analogue scale; PD-Q: pain DETECT-Questionnaire; LANSS: Leeds assessment of neuropathic symptoms and Signs.

these questionnaires were well accepted by the patient even when readministered after 24/48 hours, with a good patients' reading comprehension and ability to answer.

In particular, the good psychometric properties of the original version have been confirmed for both questionnaires, with a consistent capacity to distinguish between nociceptive and NeP. Moreover, both LANSS and PD-Q were shown to be extremely stable at test-retest analysis, a psychometric property not assessed in the original reference studies [3, 4].

This study confirmed the validity and reliability of these questionnaires for comprehensive $\mathrm{NeP}$ assessment.

For reliability, LANSS internal consistency was determined as acceptable with a Cronbach's- $\alpha$ of 0.76 in line to the result demonstrated in the original study (Cronbach's- $\alpha$ of 0.74) [4], while a lower Cronbach's- $\alpha$ value (0.65) was shown by Batistaki et al. in the Greek LANSS validation study [16].

For PD-Q, there was a good internal consistency with Cronbach's- $\alpha$ value of 0.80 , and the observed value was similar to that observed in the original study (7 Likert items, Cronbach's- $\alpha$ value: 0.83 ) [3] and in the Spanish PD-Q version (whole scale Cronbach's- $\alpha$ value of 0.86 ; 7 Likert items, Cronbach's- $\alpha$ value of 0.89 , respectively) [17]. Two questionnaires confirmed the strong ability to distinguish between nociceptive and $\mathrm{NeP}$, and to identify the neuropathic component in painful conditions with similar results showed at the retest.

Our results demonstrated a good test-retest reliability for LANSS (ICC 0.76). The original LANSS version did not assess test-retest reliability [4], and our findings are similar to those of Batistaki et al. that showed a strong correlation between the two evaluations $(r=0.94, p<0.001)$ in Greek translation of LANSS [16].

Similarly, for PD-Q, we found a high test-retest reliability (ICC 0.96 , range $0.84-0.96, p<0.05$ ); this data was not included in the original study, conducted on patient with low-back pain, considered of limited utility on estimation of pain measure reliability [3].

For interrater reliability, there was a good concordance rate $(83.3 \%)$ between reference diagnosis and LANSS (Cohen's kappa $=0.67$, CI 95\% 0.52-0.75), consistent with the original study (Cohen's kappa $=0.65$ ) [4] and with the Korean (Cohen's kappa $=0.69)[18]$ and Spanish LANSS validation study (Cohen's kappa $=0.70$ ) [8].

For the accuracy assessment, using as cutoff a value $\geq 12$ [9], LANSS confirmed an acceptable sensitivity and lower specificity ( $87 \%$ and $72 \%$, respectively) with a PPV of $83 \%$ and NPV of $79 \%$ compared to results of Bennet (sensitivity $85 \%$, specificity $80 \%$, PPV $81 \%$, NPV $84 \%$ ) [4]. In terms of convergent validity, all LANSS items showed a moderate to strong correlation with allodynia, which would be considered a strong association for a factor analysis.

Furthermore, PD-Q investigates peculiar details of gradation of descriptors, pain course pattern, and radiating pain. For example, for the sensory descriptors, $\mathrm{NeP}$ group had an intensity distribution mainly between moderate and strong, particularly for "temperature evoked pain" and "numbness sensation," defined as absent or mostly "hardly noticed" in the nociceptive group. Instead, regarding pain course pattern, we obtained similar results in both groups. No patient referred radiating distribution in other body regions. Moreover, we reported that, in $\mathrm{NeP}$ group, our translated version of PD-Q was 


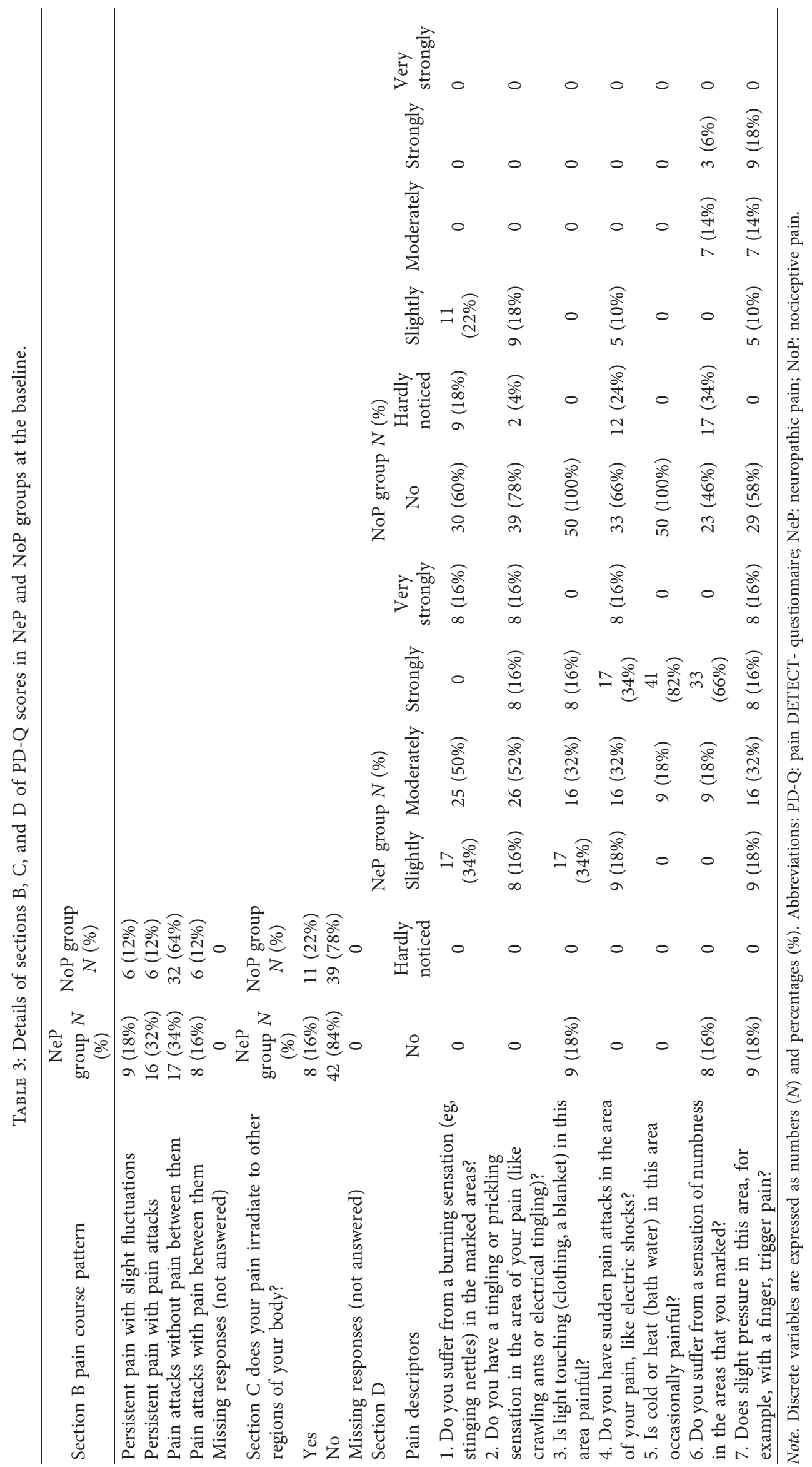


able to identify the pain course pattern specific of the neuropathic syndromes included in our study (trigeminal neuralgia and postherpetic pain) according to descriptors found in literature $[10,11]$.

Finally, the good concordance rate between the reference diagnosis and the mean value of LANSS confirmed that our translated version of the questionnaire may be considered a useful screening tool in daily clinical routine and in the therapeutic management of this insidious kind of pain.

As expected, using a unidimensional scale such as VAS, at the T0, we observed similar results in the two groups in terms of pain intensity $(p>0.05)$. This result suggests that a specialized approach to pain is strongly advised to discriminate between different pain phenotypes and also to consider several aspects of painful experience, particularly when chronic pain occurs.

Any disease associated to chronic pain, i.e., OA or fibromyalgia, results in temporal, spatial, and threshold modification of pain perception with consequent uncoupling between central (somatosensory system) and peripheral (nociceptors) activity, also known as central sensitization [7, 12]. This process explains how patients suffering from OA-related pain, typically described as nociceptive pain, could experience painful episodes with neuropathic features [7], and a multidisciplinary integrated approach is strongly suggested [13].

In a study that evaluated Self-Complete LANSS (SLANSS) and PD-Q in patients with knee OA, Moreton et al. demonstrated that PD-Q is a good surrogate measure of augmented central pain processing, while it is still debated which questionnaire between S-LANSS and PD-Q is most accurate to discriminate neuropathic pain mechanisms in knee OA [19].

In addition, LANSS successfully detects neuropathic features also in rare painful conditions such as Complex Regional Pain Syndrome type I (CRPS I) [20]. This condition typically occurs after trauma or surgery, characterized by pain out of proportion compared with the severity of the inciting event [21] without a nerve injury. Moreover, CRPS I has a relevant impact in terms of disability and quality of life and often has an unpredictable clinical course [22], so an easy-to-use tool such as LANSS may be helpful in monitoring pain changes over time in this condition [23].

A limitation of our study could be that it was performed in a clinical setting, reducing generalizability of the results.

\section{Conclusion}

This study is the first validation of Italian versions of LANSS and PD-Q. Our results demonstrated good psychometric and discriminant pain features of the translated questionnaires, with appropriate patient comprehension. Moreover, our study provides more information about the test-retest reliability, not available in original validation studies.

Reliable pain assessment, including the identification of neuropathic features, is critical to plan an appropriate therapeutic strategy. This study confirms the validity of LANSS and PD-Q as screening tools for NeP symptoms, thus improving clinical judgment in the pain management.

\section{Data Availability}

The data are available from Prof. Migliore upon reasonable request.

\section{Conflicts of Interest}

The authors declare no conflicts of interest.

\section{References}

[1] R.-D. Treede, T. S. Jensen, J. N. Campbell et al., "Neuropathic pain: redefinition and a grading system for clinical and research purposes," Neurology, vol. 70, no. 18, pp. 1630-1635, 2008.

[2] N. Torrance, B. H. Smith, M. I. Bennett, and A. J. Lee, "The epidemiology of chronic pain of predominantly neuropathic origin. results from a general population survey," The Journal of Pain, vol. 7, no. 4, pp. 281-289, 2006.

[3] R. Freynhagen, R. Baron, U. Gockel, and T. R. Tölle, "Pain DETECT: a new screening questionnaire to identify neuropathic components in patients with back pain," Current Medical Research and Opinion, vol. 22, no. 10, pp. 1911-1920, 2006.

[4] M. Bennett, "The LANSS pain scale: the leeds assessment of neuropathic symptoms and signs," Pain, vol. 92, no. 1-2, pp. 147-157, 2001.

[5] D. E. Beaton, C. Bombardier, F. Guillemin, and M. B. Ferraz, "Guidelines for the process of cross-cultural adaptation of self-report measures," Spine (Phila Pa 1976), vol. 25, no. 24, pp. 3186-3191, 2000.

[6] R. Altman, E. Asch, D. Bloch et al., "Development of criteria for the classification and reporting of osteoarthritis: classification of osteoarthritis of the knee," Arthritis \& Rheumatism, vol. 29, no. 8, pp. 1039-1049, 1986.

[7] J. R. Hochman, L. Gagliese, A. M. Davis, and G. A. Hawker, "Neuropathic pain symptoms in a community knee OA cohort," Osteoarthritis and Cartilage, vol. 19, no. 6, pp. 647-654, 2011.

[8] C. Pérez, R. Gálvez, J. Insausti, M. Bennett, M. Ruiz, and J. Rejas, "Adaptación lingüística y validación al castellano de la escala LANSS (leeds assessment of neuropathic symptoms and signs) para el diagnóstico diferencial del dolor neuropático," Medicina Clínica, vol. 127, no. 13, pp. 485-491, 2006.

[9] J. Hardy, S. Quinn, B. Fazekas, M. Agar, and D. Currow, "Can the LANSS scale be used to classify pain in chronic cancer pain trials?" Supportive Care in Cancer, vol. 21, no. 12, pp. 3387-3391, 2013.

[10] G. R. Hadley, J. A. Gayle, J. Ripoll et al., "Erratum to: Postherpetic neuralgia: a review," Erratum in: Current Pain and Headache Reports, vol. 20, no. 4, p. 28, 2016.

[11] M. Pihut, M. Szuta, E. Ferendiuk, and D. ZeńczakWięckiewicz, "Differential diagnostics of pain in the course of trigeminal neuralgia and temporomandibular joint dysfunction," BioMed Research International, vol. 2014, Article ID 563786, 7 pages, 2014.

[12] A. Latremoliere and C. J. Woolf, "Central sensitization: a generator of pain hypersensitivity by central neural plasticity," The Journal of Pain, vol. 10, no. 9, pp. 895-926, 2009.

[13] G. Iolascon, C. Ruggiero, P. Fiore, G. L. Mauro, B. Moretti, and U. Tarantino, "Multidisciplinary integrated approach for older adults with symptomatic osteoarthritis: SIMFER and SIGUIDA joint position statement," European Journal of 
Physical and Rehabilitation Medicine, vol. 56, no. 1, pp. 112-119, 2020.

[14] L. J. Cronbach, "Coefficient alpha and the internal structure of tests," Psychometrika, vol. 16, no. 3, pp. 297-334, 1951.

[15] C. B. Terwee, S. D. M. Bot, M. R. de Boer et al., "Quality criteria were proposed for measurement properties of health status questionnaires," Journal of Clinical Epidemiology, vol. 60 , no. 1, pp. 34-42, 2007.

[16] C. Batistaki, G. Lyrakos, K. Drachtidi, G. Stamatiou, M.-C. Kitsou, and G. Kostopanagiotou, "Translation, cultural adaptation, and validation of leeds assessment of neuropathic symptoms and signs (LANSS) and self-complete leeds assessment of neuropathic symptoms and signs (S-LANSS) questionnaires into the greek language," Pain Practice, vol. 16, no. 5, pp. 552-564, 2016.

[17] J. De Andrés, J. Pérez-Cajaraville, M. D. Lopez-Alarcón et al., "Cultural adaptation and validation of the painDETECT scale into Spanish," The Clinical Journal of Pain, vol. 28, no. 3, pp. 243-253, 2012.

[18] C. Park, Y.-W. Lee, D. M. Yoon, D. W. Kim, D. J. Nam, and D.-H. Kim, "Cross-cultural adaptation and linguistic validation of the Korean version of the leeds assessment of neuropathic symptoms and signs pain scale," Journal of Korean Medical Science, vol. 30, no. 9, pp. 1334-1339, 2015.

[19] B. J. Moreton, V. Tew, R. das Nair, M. Wheeler, D. A. Walsh, and N. B. Lincoln, "Pain phenotype in patients with knee osteoarthritis: classification and measurement properties of painDETECT and self-report leeds assessment of neuropathic symptoms and signs scale in a cross-sectional study," Arthritis Care \& Research, vol. 67, no. 4, pp. 519-528, 2015.

[20] P. Schestatsky, V. Félix-Torres, M. L. Fagundes Chaves et al., "Brazilian Portuguese validation of the leeds assessment of neuropathic symptoms and signs for patients with chronic pain," Pain Medicine, vol. 12, no. 10, pp. 1544-1550, 2011.

[21] M. Reimer, T. Rempe, C. Diedrichs, R. Baron, and J. Gierthmühlen, "Sensitization of the nociceptive system in complex regional pain syndrome," PLoS One, vol. 11, no. 5, Article ID e0154553, 2016.

[22] G. Iolascon and A. Moretti, "Pharmacotherapeutic options for complex regional pain syndrome," Expert Opinion on Pharmacotherapy, vol. 20, no. 11, pp. 1377-1386, 2019.

[23] A. Pepper, W. Li, W. S. Kingery, M. S. Angst, C. M. Curtin, and J. D. Clark, "Changes resembling complex regional pain syndrome following surgery and immobilization," The Journal of Pain, vol. 14, no. 5, pp. 516-524, 2013. 